\title{
The elastic analysis of cellular structures containing web openings
}

\author{
H. R. EVANS \& N. E. SHANMUGAM
}

Mr R. Sen, Bridges Engineering (Design Standards) Division, Department of Transport

The graphs presented in the Paper for determining effective shear and torsional areas will assist considerably in the analysis and design of cellular structures.

60. The use of concepts such as effective width, effective shear area and effective torsional area are essentially attempts to reproduce the threedimensional behaviour of cellular structures in a two-dimensional analysis. Consequently, it is important to bear in mind their limitations, so that weaknesses of such analytical models are recognized and corrective measures taken when needed.

61. As the Authors have explained, the shear area facility provides a means of modelling the increased flexibility of cellular structures due to openings in the cross-section or in the web. In the two-dimensional grillage model, the use of the shear area facility leads to an increase in the computed deflexion over that for an identical analysis where shear area is not considered. Unfortunately, this increase in deflexion does not result in additional bending stresses. This is because the increase in deflexion due to shear is a linear function of span and therefore does not affect the curvature or the bending moments. For the cellular structure, however, the increase in deflexion is due to bending of the cell flanges.

62. The implication of $\S 4$ is that where openings are sufficiently large as to make the flanges of cellular structures flexible, there is a likelihood that a twodimensional grillage model incorporating the shear area facility will underestimate bending stresses in the direction with the greater openings. However, the overall deflexions will be accurate.

63. For cellular bridge decks, the openings in the cross-section are generally larger and consequently the transverse bending stresses will tend to be underestimated.

64. The conclusions outlined in $\$ 5$ may be verified by reference to Figs 12 and 23. For a solid web the deflexions from the grillage analysis agree almost exactly with three-dimensional finite element solution but the maximum transverse stresses are underestimated. The results for the cases with web openings of $12.5 \%$ and $31.25 \%$ do not bear this out. However, I suspect that the effective shear coefficient $K_{\mathrm{s}}$ may have been incorrectly computed. By reference to Table 1 it

Paper published: Proc. Instn Civ. Engrs, Part 2, 1979, 67, Dec., 1035-1063. 
may be seen that $K_{\mathrm{s}}$ has been calculated on the basis of shear deflexions of $0 \cdot 24$, 0.64 and 1.526 (first series) instead of $0.095,0.495$ and 1.381 as it should be if $K_{\mathrm{s}}$ is to be unity for no openings in the web. If $K_{\mathrm{s}}$ is recalculated, it is anticipated that results for these cases will also bear out the correctness of my conclusions.

65. It is possible to adjust the transverse stresses obtained from the grillage analysis by taking into account the accuracy of its deflexions. Stresses due to the relative settlement of webs are calculated and added to the grillage results. This approach was used in the examples in reference 7 and has the advantage that the transverse bending stresses are not underestimated.

\section{Dr Evans and Dr Shanmugam}

In the idealization of cellular structures into skeletal structures, such as grillages, the shear deflexion can be expressed in terms of effective shear area, as explained in reference 1. In this study, the effective shear area was assumed to be equal to the cross-sectional area of the web and the shear deflexion was found to have little influence on the bending stresses developed within the grillage beam elements.

67. The presence of openings in the webs results in a further increase in deflexion. In this case, removal of material near the neutral axis does not significantly affect the bending deflexions. The increase in deflexions is due primarily to the increase in web shear flexibility and, in the case of large openings, vierendeel action. In the proposed method these two effects are combined and represented by the single reduced effective shear area coefficient $K_{\mathrm{s}}$.

68. The values of $K_{\mathrm{s}}$ in the Paper were calculated on the basis of this definition of $K_{\mathrm{s}}(\$ \$ 6,19$ and 20$)$. There are no errors in the computations. There may have been misunderstanding of the definition of $K_{\mathrm{s}}$; $\mathrm{Mr}$ Sen seems to suggest that there are no shear deflexions in girders without web openings.

69. Misunderstanding may have arisen from the fact that, for some girders, even when there are no openings, the value of $K_{\mathrm{s}}$ obtained from the proposed procedure does not equal unity.

70. For example, for the girder without openings in series 1 of Table 1 , the total deflexion $\delta$ obtained from the finite element method is $0.602 \mathrm{~mm}$. The bending deflexion $\delta_{\mathrm{b}}$ was obtained from the elementary formula (\$19) as $0.457 \mathrm{~mm}$. In accordance with the definition, the deflexion due to shear $\left(\delta_{\mathrm{s}}=\delta-\delta_{\mathrm{b}}\right)$ is $0.145 \mathrm{~mm}$. Therefore, from the elementary formula for shear deflexion $(\$ 20)$ the effective shear area would be obtained as $1213 \mathrm{~mm}^{2}$ with a corresponding $K_{\mathrm{s}}$ value of 0.86 . However, the value of $K_{\mathrm{s}}$ for solid webs should be 1.0 , as assumed in the Paper.

71. The difference between the assumed and calculated values of $K_{\mathrm{s}}$ in this case is due to the fact that elementary formulae are used to predict the behaviour of a complex structure. The value of $K_{\mathrm{s}}$ for the girder without web openings in series 3 of Table 1 is exactly equal to $1 \cdot 0$, thereby proving the accuracy of the formulae for longer girders.

72. When the web openings are very large, as in Figs 12 and 23 , the additional stresses resulting from the vierendeel action can be obtained by considering the isolated web, as explained in reference 2, and superimposing the local stresses on the overall stresses obtained from the grillage analysis.

73. We agree with $\mathrm{Mr}$ Sen that it is important to realize the limitations of any approximate method of analysis. The grillage idealization provides a power- 
ful and convenient method for the analysis of cellular structures. As its idealizations are based on a physical appreciation of the structural action, its limitations should be more apparent than in the case of techniques based on numerical approximations.

\section{Reference}

7. Highway Engineering Computer Branch, User Guide: slab and pseudo-slab bridge decks, Department of Transport, 1978, HECB/BI/7. 